\title{
Factors Associated with Utilization of Primary Preventive Services of Tamang Serbisyo para sa Kalusugan ng Pamilya (TSeKaP) among PhilHealth Indigent Members in Manila
}

\author{
Allan John R. Barcena, ${ }^{1}$ Carl Robert A. Casimiro, ${ }^{1}$ Gil Dominic R. Catalan, ${ }^{1}$ Camille A. Pestano, ${ }^{1}$ \\ Fenny Mariechit Q. Quinto ${ }^{1}$ and Buenalyn Teresita M. Ramos-Mortel ${ }^{2}$ \\ ${ }^{1}$ College of Public Health, University of the Philippines Manila \\ ${ }^{2}$ Department of Health Promotion and Education, College of Public Health, University of the Philippines Manila
}

\begin{abstract}
Background. The primary preventive services of TSeKaP promote disease prevention among marginalized groups. The threat of non-utilization, however, undermines the effective implementation of the program. To fill in the knowledge gap regarding utilization of primary preventive services, rate of utilization and factors associated with utilization of primary preventive services must be determined.
\end{abstract}

Objective. To determine the rate of utilization and the association of selected factors with utilization of primary preventive services of TSeKaP among PhilHealth Indigent Member of Manila.

Methods. Using a two-stage cluster sampling, 145 PhilHealth Indigent Members were randomly selected and interviewed. Chi-square test and Fisher's exact test were used to determine associations. FGDs were also conducted to gather reasons related to utilization.

Results and Conclusion. Among those surveyed, $68.28 \%$ were found to have utilized at least one primary preventive service. Age ( $p$-value $=0.021)$, knowledge ( $p$-value $=0.015)$, familiarity $(p$-value $=0.029)$, and accessibility $(p$-value $=0.013$ ) were found to be associated with utilization. Utilization generally increases as age group increases. Those with satisfactory knowledge on TSeKaP, those familiar with their health center, and those who report that their health center is accessible were 1.45 times, 2.12 times and 2.83 times more likely to utilize than their counterpart, respectively. Results showing a significant proportion of non-utilization emphasize the need to improve implementation strategies by improving not only the knowledge of respondents' on TSeKaP but also their familiarity with their health centers. The use of various communication channels, such as barangay bulletin, public address system, radio or television, may aid the dissemination of pertinent information regarding the program as perceived by the respondents.

Key Words: Health Promotion, Preventive Health Services

\section{INTRODUCTION}

Poster presented at the 2017 International Health Policy Conference, London School of Economics and Political Science, February 16 - 19, 2017, London, United Kingdom

Corresponding Author: Buenalyn Teresita Ramos-Mortel, MPH Department of Health Promotion and Education College of Public Health

University of the Philippines Manila

625 Pedro Gil Street, Ermita Manila 1000 Philippines

Telephone: +6325260811

Email: bmramosmortel@up.edu.ph
The government launched the PhilHealth Indigent Membership Program to ensure that the poorest of the poor will have access to medical care. ${ }^{1}$ PhilHealth Indigent Members are entitled to various services, including the Primary Care Benefit (PCB) Package. The PCB Package has been expanded and rebranded to Tamang Serbisyo para sa Kalusugan ng Pamilya (TSeKaP). Currently TSeKaP provides three sets of services, namely diagnostic services, covered drugs, and primary preventive services. ${ }^{2}$ 
Table 1. Prescribed frequency of utilization of primary preventive service components per member

\begin{tabular}{llc}
\multicolumn{1}{c}{ Primary Preventive Service } & \multicolumn{1}{c}{ Target Clients } & Minimum Frequency \\
Consultation & All members and dependents & Once a year \\
BP measurement & Non-hypertensive, 18 years old and above & Once a year \\
Breast examination & Hypertensive, with BP $>/=140 / 90$ & Once a month \\
Visual inspection with acetic acid & Female, 25 years old and above & Once a year \\
Body measurement & Female, 25-55 years old with intact uterus & Once a year \\
Oral check-up and prophylaxis & All members and dependents & Once a year \\
\hline
\end{tabular}

Source: TAMANG SAGOT PhilHealth Circular No. 002 - 2015 Governing Policies on the Expanded Coverage of the Primary Care Benefit Package: Tamang Serbisyo sa Kalusugan ng Pamilya (TSeKaP). http://www.PhilHealth.gov.ph/circulars/2015/TS_circ02_2015.pdf (accessed 17 November 2015).

This study is focused on primary preventive services of TSeKaP, which include consultation, body measurement, blood pressure measurement, dental service, breast examination, and cervical examination through visual inspection with acetic acid. ${ }^{2}$ These services offer a sustainable approach to health by providing cost-effective interventions for prevention and early detection of disease among Indigent Members. ${ }^{3}$ Table 1 shows the prescribed frequency of utilization of primary preventive service components per member.

Unfortunately, the threat of non-utilization undermines the goal to effectively prevent the occurrence of diseases among Indigent Members. A midterm update of the Philippine Development Plan 2011-2016 by the National Economic and Development Authority (NEDA) in 2014 states that Indigent Members who usually have low understanding of their benefits are unable to fully utilize health care services. ${ }^{4}$ In a study conducted in 2013, lack of knowledge and income group were also found to be potential barriers to utilization of social health insurance. ${ }^{5}$ Currently, there is no data regarding utilization of primary preventive services as most studies on utilization of health insurance have been limited to inpatient benefits.

This study aimed to fill in the knowledge gap by determining the rate of utilization of primary preventive services of TSeKaP among PhilHealth Indigent Members. Moreover, this study aimed to determine the association of selected factors with utilization of primary preventive services of TSeKaP. Understanding of the factors associated with utilization will provide a basis for improvement of the current health promotion and education strategies to ensure optimum utilization of the primary preventive services of TSeKaP. As the information obtained from the study contributes to the elucidation of how several factors associate with utilization, it will be valuable in providing local data to support the endeavors of PhilHealth and Manila City Health Department in providing preventive care to PhilHealth Indigent Members.

\section{METHODS}

\section{Research Design}

The study employed an analytical cross-sectional design to determine the association of several predisposing, enabling, and reinforcing factors to the utilization of the primary preventive services of $\mathrm{TSeKaP}$ among PhilHealth Indigent Members in Manila. The study employed both qualitative and quantitative tools to gather data on the utilization and nonutilization of the selected components.

\section{Study Population}

The study population is composed of listed members of PhilHealth Indigent Membership Program. These members have no visible means of income or whose income is insufficient for family subsistence. The prospective study participants were listed members from January to December 2015. Participants who refused to participate were declared as non-respondents.

\section{Sampling}

Study participants were randomly selected using a two-stage cluster sampling proportional to population size of PhilHealth Indigent Members within the barangay. Among the 6 districts of Manila, a district was chosen through simple random sampling. The chosen district is composed of zones which are barangays clustered according to geographical location. Three zones were selected through simple random sampling. All barangays from selected zones were proportionately sampled according to population size of PhilHealth Indigent Members. Through simple random sampling, Indigent Members were selected and were interviewed.

The calculation of the optimum sample size was obtained through OpenEpi using a population size of 125,000 according to Philippine National Demographic Health Survey $2013^{6}$, 95\% confidence level, 50\% anticipated utilization percent frequency and 1.5 for design effect. This yielded a minimum sample size of 144 .

\section{Operational Definition of Variables}

The dependent variable in this study is utilization of primary preventive services. Independent variables include 1) predisposing factors such as age, sex, educational attainment, knowledge on TSeKaP, and attitude towards preventive care, 2) enabling factors such as familiarity with the health facility, accessibility of health facility, and process constraints, and 3) reinforcing factors such as positive influence of family, peers, health workers, and the local government, and other groups. 
Age is the number of years that have passed since a person's date of birth. Data will be self-reported and will be confirmed through report of birthdate. It will be classified into intervals based from literature - 19-29, 30-39, 40-49, and 50 and above.

Accessibility of health facility is measured through a question on the ease of going to the health center. The health center is either accessible when the respondent answers yes, or not accessible when the respondent answers no. Other questions such as the mode of transportation, time spent, and the money spent to reach the health center are also asked to complement the answer.

Attitude towards preventive care is measured through a question on the beliefs of the respondents answered through a Likert scale.

Educational attainment is the highest level of education an individual has completed. Data will be self-reported. It will be classified into elementary, high school, and college.

Familiarity with health center is measured through a question on whether the respondent knows his or her assigned health center or not. The respondent is either familiar with the health facility when he or she answers yes, or not when he or she answers no.

Knowledge on TSeKaP is measured through a ten-point question. A score of 1-3 is classified under low knowledge, 4-6 is under satisfactory knowledge and 7-10 is high knowledge.

Process constraints are measured through a question on whether the respondents are having a problem with regard to accessing the services of the health center or not. There is either a process constraint when the respondent answers yes, or there is none when he or she answers no. A follow-up question to confirm the presence of the problem is also asked.

Reinforcing factors are measured through a question on to whom the respondents go whenever they are faced with health problems. The answer (family, peers, health workers, local government or other groups) reflects the person who influences them on their decisions regarding health. Influence may take the form of giving advice, guiding decisions or directing actions.

Sex is a person's biological status categorized as male or female. Data will be self-reported.

Utilization is defined according to the accomplishment of the respondents of the prescribed minimum frequency of utilization of primary preventive services, as enumerated in Table 1. It is classified as utilized or never utilized. A person utilized when he availed at least one primary preventive service within the prescribed minimum frequency.

\section{Data Collection}

A total of 145 members were interviewed. An interview schedule was used to collect information on their utilization of primary preventive services. After the interview, respondents were asked regarding their willingness to participate in a subsequent focus group discussion. Three FGDs composed of about 6-8 persons were held. This was conducted by a faculty of the University of the Philippines Manila and a known expert on Health Promotion and Education.

Prior to the conduct of the study, pre-testing of tools to a population group with similar characteristics as those of the target group was employed. In line with the results of pretesting, a session was conducted regarding standardization of data collection tools among the researchers.

\section{Data Analysis}

The data gathered from the interview were encoded and tabulated using Epi Info ${ }^{\mathrm{TM}}$. Utilization, which was defined as the use of at least one primary preventive service of TSeKaP according to the frequency prescribed by PhilHealth, was compared across predisposing, enabling, and reinforcing variables. To determine the association of PhilHealth utilization to different factors, chi-square test of association and Fisher's exact test were employed. Fisher's exact test was used when a cell had an expected value of less than 1 or when more than $20 \%$ of the cells had an expected value of less than 5. For the rest of the tables, chi-square test was used. The alpha used was 0.05. Data from the FGD were transcribed verbatim. FGD transcripts were summarized and themes were extracted. Data were used to supplement quantitative data.

\section{Ethical Considerations}

The study has been duly reviewed and approved by the UPM Research Ethics Board Review Panel (College of Public Health). It has been assigned the study protocol code UPMREB 2016-066-UND. Participation of the study subjects was completely voluntary. For the conduct of both interviews and focus group discussions, written consent form was obtained from the participants.

\section{Internal Validity}

To test the internal validity of the study, face validity and reliability of study instruments were tested. As part of the face validity test, the structured tools for the study were presented to three knowledgeable people to evaluate the appropriateness, suitability, and the mechanics. For reliability of the tools, the tools were pre-tested to ten indigents that were not part of the sample cluster.

\section{External Validity}

The results of the study are only applicable to the city of Manila because of the sampling design employed.

\section{RESULTS}

\section{Rate of Utilization}

A total of 145 PhilHealth Indigent Members in the $5^{\text {th }}$ district of Manila participated in the study. Among the PhilHealth Indigent Members surveyed, 68.28\% were found to have utilized at least one preventive service component of TSeKaP as shown in Table 2. 
Table 2. Rate of utilization of the respondents

\begin{tabular}{lcc} 
& Frequency & Percentage \\
Utilization & & \\
Utilized & 99 & 68.28 \\
Never utilized & 46 & 31.72 \\
\hline
\end{tabular}

Table 3 shows the utilization by age, sex, educational attainment, knowledge on TSeKaP, and attitude towards preventive care.

Table 4 shows the utilization by familiarity with health center, accessibility of health center, and process constraints.

Table 5 shows the utilization by positive influence of family, peers, health workers, local government, and other groups.

\section{Association of Selected Factors with Utilization}

Table 6 shows the association of predisposing variables with utilization. The results reveal that age $(p=0.021)$ is significantly associated with utilization. Knowledge on $\mathrm{TSeKaP}$ is also associated with utilization $(p=0.015)$. The prevalence ratio reveals that those with satisfactory knowledge on TSeKaP were 1.45 times more likely to utilize than those with low knowledge on TSeKaP.

Table 7 shows the association of enabling variables with utilization. The results reveal that familiarity with health center is significantly associated with utilization ( $p$-value $=0.029$ ). The prevalence ratio reveals that those familiar with their health center assignment were 2.12 times more likely to utilize than those who are not familiar. As to accessibility and utilization, the results show that accessibility of health center is significantly associated with utilization ( $\mathrm{p}$-value $=0.013$ ). The prevalence ratio reveals that those who report that their health centers are accessible were 2.83 times more likely to utilize than those who report that their health centers are not accessible. However, the findings show that experience of process constraints is not associated with utilization.

Table 8 shows the association of reinforcing variables with utilization. The results show that the positive influence of family is not associated with utilization. Likewise, the positive influence of peers, health workers, local government, and other groups are not associated with utilization.

Table 3. Rates of utilization according to predisposing variables [ $n=145]$

\begin{tabular}{|c|c|c|c|c|}
\hline \multirow{2}{*}{ Predisposing Variables } & \multicolumn{2}{|c|}{ Utilized } & \multicolumn{2}{|c|}{ Never utilized } \\
\hline & Frequency & Percentage & Frequency & Percentage \\
\hline \multicolumn{5}{|l|}{ Age } \\
\hline $19-29$ & 15 & 50.00 & 15 & 50.00 \\
\hline $30-39$ & 21 & 75.00 & 7 & 25.00 \\
\hline $40-49$ & 31 & 83.78 & 6 & 22.22 \\
\hline 50 and above & 32 & 64.00 & 18 & 36.00 \\
\hline \multicolumn{5}{|l|}{ Sex } \\
\hline Male & 65 & 73.86 & 23 & 26.14 \\
\hline Female & 34 & 59.65 & 23 & 40.35 \\
\hline \multicolumn{5}{|l|}{ Educational Attainment } \\
\hline Elementary & 19 & 59.38 & 13 & 40.63 \\
\hline High School & 60 & 73.17 & 22 & 26.83 \\
\hline College & 20 & 64.52 & 11 & 84.62 \\
\hline \multicolumn{5}{|l|}{ Knowledge on TSeKaP } \\
\hline Low knowledge & 83 & 64.84 & 45 & 35.16 \\
\hline Satisfactory knowledge & 16 & 94.12 & 1 & 94.12 \\
\hline High knowledge & 0 & 0.00 & 3 & 0.00 \\
\hline \multicolumn{5}{|l|}{ Attitude towards Preventive Care } \\
\hline Negative & 2 & 66.67 & 1 & 33.33 \\
\hline Neutral & 1 & 100.00 & 0 & 0.00 \\
\hline Positive & 45 & 31.91 & 96 & 68.09 \\
\hline
\end{tabular}

Table 4. Rates of utilization according to enabling variables

\begin{tabular}{|c|c|c|c|c|}
\hline \multirow{2}{*}{ Enabling Variables } & \multicolumn{2}{|c|}{ Utilized } & \multicolumn{2}{|c|}{ Never utilized } \\
\hline & Frequency & Percentage & Frequency & Percentage \\
\hline \multicolumn{5}{|l|}{ Familiarity with Health Center } \\
\hline Familiar & 96 & 70.59 & 40 & 29.41 \\
\hline Not familiar & 3 & 33.33 & 6 & 66.67 \\
\hline \multicolumn{5}{|l|}{ Accessibility of Health Center } \\
\hline Accessible & 97 & 70.80 & 40 & 29.20 \\
\hline Not accessible & 2 & 25.00 & 6 & 75.00 \\
\hline \multicolumn{5}{|l|}{ Process Constraints } \\
\hline Encountered & 22 & 78.57 & 6 & 21.43 \\
\hline Did not encounter & 77 & 65.81 & 40 & 34.19 \\
\hline
\end{tabular}


Table 5. Rates of utilization according to reinforcing variables

\begin{tabular}{|c|c|c|c|c|}
\hline \multirow{2}{*}{ Reinforcing Variables } & \multicolumn{2}{|c|}{ Utilized } & \multicolumn{2}{|c|}{ Never utilized } \\
\hline & Frequency & Percentage & Frequency & Percentage \\
\hline \multicolumn{5}{|l|}{ Family } \\
\hline Influenced & 67 & 68.37 & 31 & 31.63 \\
\hline Not influenced & 32 & 68.09 & 15 & 31.91 \\
\hline \multicolumn{5}{|l|}{ Peers } \\
\hline Influenced & 14 & 77.78 & 4 & 22.22 \\
\hline Not influenced & 85 & 66.93 & 42 & 33.07 \\
\hline \multicolumn{5}{|l|}{ Health Workers } \\
\hline Influenced & 35 & 68.63 & 16 & 31.37 \\
\hline Not influenced & 64 & 68.09 & 30 & 31.91 \\
\hline \multicolumn{5}{|l|}{ Local Government } \\
\hline Influenced & 5 & 71.43 & 2 & 28.57 \\
\hline Not influenced & 94 & 68.12 & 44 & 31.88 \\
\hline \multicolumn{5}{|l|}{ Other groups } \\
\hline Influenced & 3 & 75.00 & 1 & 25.00 \\
\hline Not influenced & 96 & 68.09 & 45 & 31.91 \\
\hline
\end{tabular}

Table 6. Association of predisposing variables with utilization

\begin{tabular}{ll}
\multicolumn{1}{c}{ Predisposing Variables } & p-value \\
Age & $0.021^{*}$ \\
Sex & 0.072 \\
Educational Attainment & 0.320 \\
Knowledge on TSeKaP & $0.015^{*}$ \\
Attitude towards Preventive Care & 0.790 \\
\hline
\end{tabular}

${ }^{*}$ AT $\alpha=0.05$

Table 7. Association of enabling variables with utilization

\begin{tabular}{ll}
\multicolumn{1}{c}{ Enabling Variables } & p-value \\
Familiarity with Health Center & $0.029^{*}$ \\
Accessibility of Health Center & $0.013^{*}$ \\
Process Constraints & 0.193 \\
\hline
\end{tabular}

${ }^{*}$ AT $\alpha=0.05$

Table 8. Association of reinforcing variables with utilization

\begin{tabular}{lc}
\multicolumn{1}{c}{ Reinforcing Variables } & p-value \\
Family & 0.973 \\
Peers & 0.355 \\
Health Workers & 0.947 \\
Local Government & 1.000 \\
Other groups & 1.000 \\
\hline
\end{tabular}

${ }^{*}$ AT $\alpha=0.05$

\section{Brief Narrative of Focus Group Discussions}

The FGD participants are all utilizers of at least one primary preventive service. Their ages range from 30 to 74 years old and most of them are female. None of the participants knew what the acronym TSeKaP stands for. When asked to define or describe TSeKaP, some participants described the routine "check-up" instead of the PhilHealth program. However, it was notable that some participants described TSeKaP as a program for disease prevention. Most of the participants reported that they only consult when they feel symptoms already. One participant mentioned that it is necessary to avail TSeKaP even when there are no symptoms felt. Despite this belief, she reported that she herself is not able to do so.
The participants describe the service being offered by the health center as generally good. However, they have enumerated several issues. Some participants report that they are discouraged by long queues, congestion, and daily quotas. Also, most of the participants reported that some health workers allow some patients to bypass the line. Some participants reported that health workers are sometimes not accommodating and are indifferent towards indigents. Other reasons that discourage participants from consulting their respective health centers include lack of immediate incentives, lack of medicine and supplies, and fear of knowing whether they are ill or not.

Most of the participants prefer to have their weight measured in alternative centers because health centers are often crowded. It is also important to note that most pregnant mothers coincidentally availed consultation and weight measurement alongside their general pregnancy check-up. Although most participants monitor their blood pressure, they prefer to avail the services offered for free or for a few pesos by nearby clinics. Those who are not hypertensive feel that there is no need to monitor their blood pressure. All of the participants who availed the dental examination coincidentally availed the service prior to a tooth extraction. However, none of the participants went to the center for dental check-up alone. Participants prefer dental missions offered by different organizations and dental clinics more proximate to their households.

No one had a breast examination in the health center before. The participants are unaware that breast examination is offered by the health center. The participants also added that they do not feel the need for a breast examination because they do not feel any symptoms. The participants' unawareness of the visual inspection of cervix translates to non-utilization. Remarkably, only one tried the service before. However, she was discouraged to get her results since it took her a very long time following up. Similarly, the participants added that they do not feel the need for a visual inspection of cervix because they do not feel any symptoms. 
When the participants were asked for suggestions on how the TSeKaP program would better reach them, all proposed that it would be helpful to have announcements in their respective barangays either through the barangay bulletin, public address system, radio or television.

The following themes were identified from the focus group discussions:

1. Most of the respondents are not aware of the TSeKaP as a program.

2. Most of the FGD participants visit the health center only when they are ailing.

3. Some utilizers of TSeKaP did not purposively utilize the program but incidentally availed it due to overlapping programs.

4. The lack of awareness on the specific services under TSeKaP resulted to non-utilization of these services.

5. The respondents wish that there will be announcements on TSeKaP for them to be made aware of their benefits.

\section{DISCUSSION}

While the majority of the respondents (68.28\%) reported utilization of primary preventive services, the proportion of the respondents that have never utilized is still considerable given that this service is supposed to reach out to every indigent family.

\section{Predisposing Variables}

Results of the test of association revealed that age and knowledge on TSeKaP were significantly associated with utilization.

For age, the '30-39' age group tends to utilize primary preventive services of TSeKaP 1.5 times more likely than the base group which is ' $19-29$ '. The ' $40-49$ ' age group tends to utilize 1.68 times more likely than the base group, and the ' 50 and above' age group tends to utilize 1.28 times more likely than the base group. These are in line with the study by Ladwig et al. (2000) showing that utilization increases as the age group increases as individuals are predisposed to gain increased susceptibility to diseases and tend to realize the value of their health as they age. ${ }^{7}$ While the utilization of those who belong to the ' 50 and above' age group is significantly higher than the base group, it did not follow the trend of being higher than the previous age group. This may be attributed to the reduction in mobility among the age group which significantly limits their utilization of the preventive services when compared to other age groups higher than the base.

The results show that those with satisfactory knowledge were 1.45 times more likely to utilize than those with low knowledge on TSeKaP. According to the study by Quimbo et al., having more extensive knowledge on the benefits predisposes the members to maximize them. ${ }^{8}$ The data from the FGD shows that indeed the lack of knowledge has hindered the participants to avail all of the services offered by TSeKaP. While some incidentally utilized the services due to overlapping programs such as the Well Baby program, most of the participants reported that they did not know TSeKaP exists and that services, specifically the breast and cervical exams are thought to be not available in the health center.

Educational attainment was found to be not associated with utilization. According to the study of Quimbo et al., educational attainment of the household head is a statistically significant factor in PhilHealth utilization, that underutilization of PhilHealth benefits for hospitalization is negatively associated with the mother's educational attainment. ${ }^{8}$ However, in this study, no such pattern was observed. Paying members are expected to utilize more as they expect equal value for what they pay. ${ }^{9}$ For indigent members who belong to the lower income bracket and avail the primary preventive services for free, this may not be the case. The association of utilization with knowledge and not with educational attainment suggests that the utilization rates among indigent PhilHealth members can be influenced by providing information through other pathways.

Attitude was found to be not associated with utilization. It was found out that majority of the participants had a positive attitude whether they utilized TSeKaP or have never utilized it at all. The positive attitude not translating into practice can be explained by Kelman's three processes of social influence. It states that the translation of attitude to practice can be distinguished into three processes, which are compliance, identification and internalization. It may be the case that the participants are caught at the compliance stage and are heavily reliant on the command of an authority. Thus, participants would opt to not consult their health center unless someone from a position of authority says so.

\section{Enabling Variables}

Results of the test of association revealed that familiarity with health center ( $p$-value $=0.029$ ) and accessibility of the health center ( $p$-value $=0.013$ ) were found to be significantly associated with utilization.

Respondents who were familiar with their health center assignment were 2.12 times more likely to utilize than those who are not familiar. Individuals unfamiliar with their health center assignment are more likely to look for alternative sources of service thereby decreasing the chances of that individual accessing the service of the health center. Furthermore, those who found their health centers accessible were 2.83 times more likely to utilize than those report that their health centers are not accessible. According to the study by Field and Briggs, (2001) barriers to effective accessibility such as distance, inconvenience and cost may deter and reduce utilization of health care. ${ }^{10}$ Thus, the riddance from these inconveniences yields to accessibility and consequently, to utilization of health care in general.

Process constraints were found not to be associated with utilization. This may be attributed to how the participants 
perceive these constraints. The participants do not see the difficulties they encounter whenever they go to their respective health center as constraints. Most of them understand that irregularities normally arise from the provision of public services. Thus, in order to avail the free services offered by the health center, they are willing to go through extents of difficulty.

\section{Reinforcing Variables}

Results of the test of association revealed that the positive influence of family, peers, health workers, local government, and other groups are not associated with utilization. This may be explained by the weaker relations among people in the community as many indigent members are transient dwellers and have just been relocated few years ago. Because of this, members may develop a lower sense of belongingness making it less likely for actors within the community to influence their behavior. Moreover, although most rely on family members for reinforcement, family members of indigent families usually have the same level of knowledge on TSeKaP which may not be enough to drive behavioral change towards utilization. It may also be possible that these family members are not always present to constantly reinforce or remind them or increase their health-seeking behavior. Furthermore, results of the FGD show that instead of acting as reinforcers, the health workers were perceived by the participants to be not accommodating towards indigents discouraging most of the participants to go back to the health center and avail of the services.

\section{CONCLUSION}

It was found out that $68.28 \%$ of the PhilHealth Indigent Members surveyed utilized at least one of the primary preventive components of TSeKaP. This provides baseline information regarding the current status of the program with respect to the primary preventive service components. Furthermore, the association of the selected factors with utilization could provide an insight as to how these factors could play a role in health promotion and education campaigns to improve utilization rates especially among target marginalized groups.

While the majority of the respondents reported utilization, a significant proportion of the respondents still have not utilized the primary preventive service. This emphasizes the need to improve current implementation strategies by improving not only the knowledge of respondents' on $\mathrm{TSeKaP}$ but also their familiarity with their health centers. The use of various communication channels, such as barangay bulletin, public address system, radio or television, may aid the dissemination of pertinent information regarding the program as perceived by the respondents.

Moreover, program implementers in cooperation with PhilHealth could incorporate health education and information dissemination regarding the services catered by the health centers to the different activities they are currently conducting.

\section{Limitations of the Study}

Information bias may have been encountered as both factors and outcome were measured at the same time-point. To minimize this, the methods used were standardized and validated, and objective measures were used to help avoid information inaccuracies or biases. Specifically, in this study, with the tool being an interview, self-reporting presented a tendency for the participants to provide what they perceived to be socially desirable answers rather than the truth, particularly when it came to behavioral aspects and health conditions associated with taboos. As such, the researchers minimized this by acting professionally and establishing trust.

\section{Acknowledgments}

This study would not have been possible without the assistance of a number of people. Firstly, the researchers would like to thank Dr. Benjamin M. Yson, the Acting Manila City Health Officer, Dr. Gina F. Pardilla, the Head of City Health Planning and Development Office, Dr. David B. Pinto, the District Health Officer of the District V Health Office, and the staff of the Manila Health Department for their valuable endorsement and insights regarding the study.

The researchers would also like to thank the officersin-charge and staff of Fabella Health Center, Paco Health Center, and San Andres Health Center for providing the lists of PhilHealth Indigent Members and the venues for the focus group discussions among selected participants.

The researchers' gratitude also goes to the Barangay Chairpersons and Barangay Council Members who provided assistance and support throughout the conduct of the study in their respective barangays.

The researcher team would also like to express utmost gratitude to its beloved adviser, Prof. Buenalyn Teresita M. Ramos-Mortel of the Department Health Promotion and Education, for her invaluable remarks and recommendations throughout the course of the study; and Dr. Abubakar Asaad for sharing his expertise on epidemiology and biostatistics which contributed to the improvement of the study's methodology and analysis.

Ultimately, the researchers would like to thank their families for providing moral and financial support to make this research possible.

\section{Statement of Authorship}

All authors have approved the final version submitted.

\section{Author Disclosure}

All authors have declared no conflict of interest.

\section{Funding Source}

No external funding. 


\section{REFERENCES}

1. Fernandez M, Brillantes Jr. A, Modino A, The National Health Insurance Program in the Philippines: Critical Challenges and Future Directions [Online]. [cited 2015 Nov]. Available from http://dirp4. pids.gov.ph/webportal/CDN/EVENTS/Fernandez_NHIPppt.pdf.

2. TAMANG SAGOT Enhancements to Primary Care Benefit 1 now called "TSEKAP" Package Starting CY 2014 [Online]. 2014 [cited 2015 Nov]. Available from http://www.philhealth.gov.ph/ circulars/2014/TS_circ15_2014.pdf.

3. Pantig, IM. Sustainability of the National Government Premium Subsidy for Indigents. Philippine Journal of Development 2013; 40.

4. National Economic and Development Authority. Philippine DevelopmentPlan2011-2016MIDTERMUPDATEwith Revalidated Results Matrices. Pasig City: National Economic and Development Authority [Online]. 2014 [cited 2015 Nov]. Available from http:// www.neda.gov.ph/wp-content/uploads/2014/08/NEDA-PDP-20112016-Midterm-Update+Errata2.pdf (accessed 18 November 2015).

5. Faraon E, Estrada J, Farillas E. et al. Significant Predictors of Underutilization of Inpatient Benefits among PhilHealth Members in Selected Barangays in Manila. Acta Medica Philippina. 2013; 47(3): 69-73.
6. Philippine Statistics Authority. Philippines National Demographic and Health Survey 2013 (Online). (Cited 2015 Nov.) Available from https://dhsprogram.com/pubs/pdf/FR294/FR294.pdf

7. Ladwig KH, Marten-Mittag B, Formanek B, Dammann G. Gender differences of symptom reporting and medical health care utilization in the German population. European Journal of Epidemiology. 2000; 16: 511-18.

8. Quimbo S, Florentino J, Peabody JW, Shimkhada R, Panelo C, Solon $\mathrm{O}$. Underutilization of social insurance among the poor: evidence from the Philippines. PloS One.; 3(10).

9. Puyat MEA, Factors Influencing PhilHealth Coverage and In-patient Benefit Utilization of Filipino Children under Five [Online]. 2013 [cited 2015 Nov]. Available from http://dirp4.pids.gov.ph/ris/dps/ pidsdps1337.pdf.

10. Field K, Briggs D. Socio-economic and locational determinants of accessibility and utilization of primary health-care. Health and Social Care in the Community. 2001; 9(5): 294-308. 